\title{
Non-Invasive Management of Natal Tooth
}

\author{
Geethanjali G. ${ }^{1}$, Amarshree A. Shetty ${ }^{2}$, Amitha M. Hegde², Delisha Y. Fernandes ${ }^{4}$
}

\begin{abstract}
${ }^{1}$ Department of Pedodontics and Preventive Dentistry, A. B. Shetty Memorial Institute of Dental Sciences, NITTE (Deemed to be University), Mangalore, Karnataka, India. ${ }^{2}$ Department of Pedodontics and Preventive Dentistry, A. B. Shetty Memorial Institute of Dental Sciences, NITTE (Deemed to be University), Mangalore, Karnataka, India. ${ }^{3}$ Department of Pedodontics and Preventive Dentistry, A. B. Shetty Memorial Institute of Dental Sciences, NITTE (Deemed to be University), Mangalore, Karnataka, India. ${ }^{4}$ Department of Pedodontics and Preventive Dentistry, A. B. Shetty Memorial Institute of Dental Sciences, NITTE (Deemed to be University), Mangalore, Karnataka, India.
\end{abstract}

\section{INTRODUCTION}

The first primary tooth erupts in the oral cavity at around 6 months of age and is one of the important milestones of oral development. But in rare cases, there may be accelerated eruption much earlier than the ideal time of eruption. Such eruption is termed as precocious eruption/ premature eruption. ${ }^{1}$ It was Massler and Savara in the year 1950, who gave a definite terminology namely natal and neonatal teeth based on the timing of eruption of these teeth. Natal teeth refers to the teeth present at the time of birth whereas neonatal teeth refers to those that erupt within the first 30 days of life. ${ }^{2}$ The incidence of natal teeth has been reported to be 1:2000 to $1: 3500 .^{3}$ This condition is of clinical importance to both the pediatrician as well as the pediatric dentist due to the complications associated with it. The frequently reported consequences include, risk of aspiration, ulceration of ventral surface of tongue thereby interfering with feeding, traumatic ulceration of the mother's nipples. These complications warrant removal of the offending tooth. But before planning the removal, the pediatric dentist should rule out if the tooth is a part of primary dentition or is an additional entity. The present case is a report of a 6 days old infant who reported with a natal tooth diagnosed at the time of birth by the pediatrician.

\section{PRESENTATION OF CASE}

A 6 days old male patient was referred to the Department of Pedodontics and Preventive Dentistry by a Pediatrician due to the presence of tooth in the anterior lower jaw at the time of birth. The tooth was diagnosed during routine pediatric evaluation after delivery. Medical history revealed that the child was a full term baby born through vaginal delivery. The child weighed 3.2 kilograms at birth and was otherwise healthy. On intra- oral examination it was found that there was a single natal tooth in the lower front tooth that was covered by soft tissue. On palpation, the tooth exhibited no mobility. Soft tissue examination revealed that the surrounding soft tissues were clinically normal with no ulceration of the ventral surface of tongue. (fig. 1) On eliciting the post- natal history, the mother reported no discomfort during feeding and breast feeding was carried out normally. Since the tooth was firm with no mobility, no surrounding soft tissue ulceration or difficulty in feeding, a clinical decision was made to not to extract the tooth and keep it under observation. The parent was informed about the same and was advised to report for a follow- up every month so as to assess the presence of any mobility or soft tissue ulceration. A follow up after 6 months revealed an uneventful outcome with the crown of the primary lower primary central incisor being clearly visible indicating that it was a premature eruption of primary tooth.
Corresponding Author:

Dr. Amarshree A. Shetty,

Department of Pedodontics \&

Preventive Dentistry,

A. B. Shetty Memorial Institute of Dental Sciences, NITTE (Deemed to be University), Mangalore-575018, Karnataka, India.

E-mail: dramarshreeshetty@gmail.com

DOI: $10.14260 /$ jemds/2020/306

Financial or Other Competing Interests: None.

How to Cite This Article:

Geethanjali G, Shetty AA, Hegde AM, et al. Non-invasive management of natal tootht. J. Evolution Med. Dent. Sci. 2020;9(16): 1402-1404 DOI:

10.14260/jemds/2020/306

Submission 16-02-2020,

Peer Review 04-04-2020,

Acceptance 10-04-2020,

Published 20-04-2020.
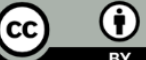

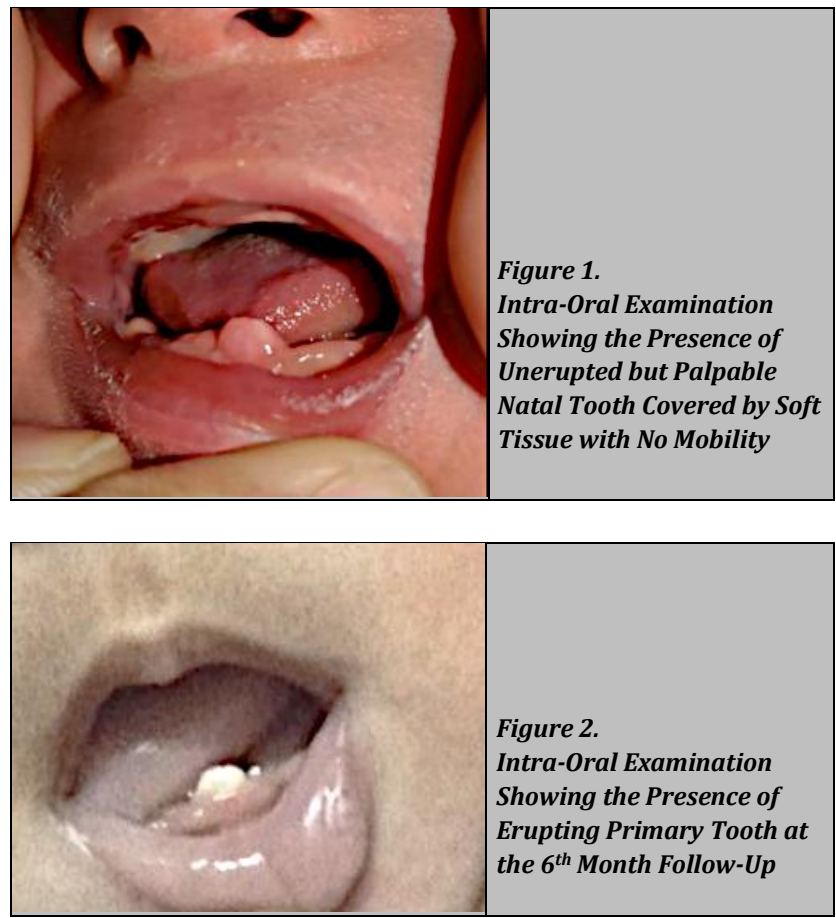

\section{DISCUSSION}

One of the earliest oral health issues that a newborn might face is anomaly of tooth eruption that manifests as natal/ neonatal tooth. The incidence is reported to be 1:2000 to 1:3500 with no gender predilection. ${ }^{4}$ The teeth that are commonly affected include: Primary mandibular central incisors $(85 \%)>$ maxillary central incisors $(11 \%)>$ mandibular canines and molars $(3 \%)>$ maxillary canines and molars $(1 \%) .^{5}$

The actual etiology is unknown, and several hypotheses have been stated that include

- Superficial position of tooth germ.

- Infection or malnutrition.

- Accelerated eruption due to febrile incidents or hormonal stimulation.

- Hereditary transmission of a dominant autosomal gene.

- Osteoblastic activity inside the germ area related to the remodeling phenomenon.

- Hypovitaminosis. ${ }^{4}$

- Mothers heavily exposed to polychlorinated biphenyls (PCBs) and dibenzofurans (PCDFs). ${ }^{6}$

Although there are no studies available to confirm a causal relationship, the superficial position of the germ associated with a hereditary factor is the most widely accepted etiology. Natal and neonatal teeth have been associated with syndromes such as Ellis-van Creveld Syndrome, Pierre Robin Syndrome, Hallermann-Streiff Syndrome, Sotos Syndrome, Cleft lip and cleft palate etc. Helbing et al, 1997, classified natal teeth into the following categories-

1. Shell-shaped crown loosely attached to the alveolus by a rim of oral mucosa without any root

2. Solid crown loosely attached to the alveolus by oral mucosa with little or no root

3. Incisal edge of crown just erupted through oral mucosa

4. A mucosal swelling with palpable but unerupted tooth. ${ }^{4}$
Natal teeth are a cause of concern due to the complications associated that include, aspiration, ulceration of ventral surface of tongue commonly called as Riga- Fede disease, difficulty in suckling and feeding which can affect the nutrition of the child. When such complications arise it is advised to extract the tooth. Such an extraction should be carried out after administration of Vitamin K (0.5-1 mg IM) since the commensal intestinal flora would not have been developed before 10 days of age.

King and Lee (1989) in their clinical study of 59 prematurely erupted teeth reported that $42.4 \%$ of teeth were extracted due to excessive mobility. The concern regarding extraction is the fact that the tooth may be a part of the primary dentition which if removed may lead to space loss, collapse of arch leading to malocclusion. So the suggested alternative treatment strategies when the tooth does not exhibit mobility include, smoothening of the incisal edges, addition of composite resin to the incisal edge, breast pump or feeding plate all of which would enable adequate nutrition to the child. ${ }^{7}$ However if the tooth is poorly developed with extreme mobility that poses a risk of aspiration then extraction is definitely indicated.

In the present case, since the tooth did not exhibit any mobility and no interference with breast feeding was noted, the clinical decision for a non- invasive management was made. However to monitor the development of complications that might arise at a later stage, a monthly follow up was advised to the parents. The follow up at 6 months revealed the presence of primary central incisor indicating that it was a premature eruption of primary tooth. If an extraction had been carried out earlier then it would have led to undesirable consequences regarding the occlusion and arch equilibrium. So the non- invasive wait and watch approach in this case proved to be effective.

\section{CONCLUSIONS}

Oral health care provided in the first year of life of a child greatly influences the future oral health practices of the child. The pediatric dentist should be aware of the possible oral health issues that might occur during the formative years of a child so as to provide appropriate infant oral health care.

\section{REFERENCES}

[1] Basavanthappa NN, Kagathur U, Basavanthappa RN, et al. Natal and neonatal teeth: a retrospective study of 15 cases. Eur J Dent 2011;5(2):168-72.

[2] Anegundi RT, Sudha P, Kaveri H, et al. Natal and neonatal teeth: a report of four cases. J Indian Soc Pedod Prev Dent 2002;20(3):86-92.

[3] Buchanan S, Jenkins CR. Riga-Fedes syndrome: natal or neonatal teeth associated with tongue ulceration. Case report. Aust Dent J 1997;42(4):225-7.

[4] Cunha RF, Boer FA, Torriani DD, et al. Natal and neonatal teeth: review of the literature. Pediatr Dent 2001;23(2):158-62.

[5] Leung AKC, Robson WLM. Natal teeth: a review. Journal of The National Medical Association 2006;98(2):226-8. 
[6] Alaluusua S, Kivirantha H, Leppaniemi A, et al. Natal and neonatal teeth in relation to environmental toxicants. Pediatr Res 2002;52(5):652-5.
[7] Singh S, Subbareddy VV, Dhananjaya G, et al. Reactive fibrous hyperplasia associated with a natal tooth: a case report. J Indian Soc Pedod Prev Dent 2004;22(4):183-6. 